\title{
DRIVING SIMULATOR ASSESSMENT OF FITNESS-TO-DRIVE FOLLOWING TRAUMATIC BRAIN INJURY
}

\author{
Benjamin McManus, Tyler R. Bell, Despina Stavrinos \\ University of Alabama at Birmingham \\ Birmingham, AL, USA \\ Email: bmcmanus@uab.edu
}

\begin{abstract}
Summary: Returning to driving is a major goal for individuals recovering from a traumatic brain injury (TBI). Clinicians have a variety of tools to assess the ability to return to driving for TBI patients, including cognitive assessments, but on-road instrumented vehicle driving assessments have been considered the gold standard. However, these on-road assessments are limited in the ability to ethically expose drivers to certain driving situations or environments. The purpose of this study was to examine the ability of a high-fidelity driving simulator to assess driving performance in individuals who have sustained a moderate-to-severe TBI, as well as associate cognitive measures commonly used in this population with simulated driving outcomes. Fourteen participants from a TBI clinic were recruited to drive in a simulator through a series of increasingly complex diving modules: 1) basic vehicle operation; 2) secondary task engagement while driving; 3) car following; 4) divided attention; and 5) navigating left hand turns across oncoming traffic. Half $(n=7)$ had been released to return to drive and half $(n=7)$ were considered to never be able to return to driving. Although general trends suggest non-drivers exhibit slower driving and increased lane position variation, group differences driving were not shown likely due to small sample sizes. Differences in patterns of cognitive correlates with driving were found, with higher order cognitive processes, like working memory, being more associated with driving outcomes in active drivers. Suggestions for driving scenario development in this population are discussed.
\end{abstract}

\section{INTRODUCTION}

Following a traumatic brain injury (TBI), driving is a key goal in establishing personal independence after recovery. TBI contributes to nearly $30 \%$ of all injury deaths in the United States, and motor vehicle collisions (MVCs) account for nearly $26 \%$ of all unintentional injury deaths (Xu, Murphy, Kochanek, \& Bastian, 2016). The overwhelming majority of MVCs are caused by driver error due to inattention, poor environment surveillance, and misjudgment of gaps and other vehicles' movements (National Highway Traffic Safety Administration, 2015). These same factors are also effects seen in post-TBI (e.g., impaired thinking, memory, movement).

For clinicians determining whether to allow individuals to return to driving, on-road evaluations have been a gold standard for assessing driving ability. However, such evaluations are limited in the ability to expose drivers to unpredictable environments, situations, or hazards often encountered in driving. As an alternative, driving simulators may provide a viable tool in informing clinical decisions on driving after TBI (Lew, Rosen, Thomander, \& Poole, 2009). Driving simulators provide the ability to safely expose drivers to situations not possible in on-road driving tests. The purpose of this pilot study was to 1) determine if a driving simulator can assess differences between active drivers and non-drivers after a moderate-to-severe TBI; and 2) identify cognitive correlates of driving in individuals who have successfully returned to driving after a 
TBI and in individuals who will likely never return to driving after a TBI. It was hypothesized that active drivers would perform significantly better than non-active drivers.

\section{METHOD}

\section{Participants}

Fourteen participants ages $20-50$ years $\left(M_{\text {age }}=33.59\right.$ years, $\left.S D=7.98\right), 64 \%$ male, and $100 \%$ Caucasian, were recruited from a TBI clinic during routine appointments and via mailed letters. Participants were categorized into to one of two groups based on the TBI clinicians' determinations of the ability to return to driving activities: 1) individuals who successfully resumed driving activities and report currently driving at least 3 times per week $(n=7)$; and 2$)$ individuals who have not been approved to return to driving after TBI and are unlikely to drive independently again $(n=7)$. Inclusion criteria for the successful drivers included 1) no at-fault crashes or moving violations in the past 3 years; and 2) no other interfering neurological and physical health issues. Participants in either group were excluded if they 1) did not possess a valid driver's license; 2) did not drive pre-TBI, or 3 ) had comorbidities impacting fitness-to-drive.

\section{Experimental Designs and Materials}

Driving Simulator. Driving was assessed in a series of driving scenarios designed as distinct modules to simulate a specific driving task, environment, or situation that increased in complexity from module-to-module via increases in visual and strategic complexity of the environment (Edquist, Horberry, Hosking, \& Johnston, 2011; Jahn, Oehme, Krems, \& Gelau, 2005) and strategic control. Participants drove in a Realtime Technologies, Inc. driving simulator outfitted with a 2016 Honda Pilot featuring fully functioning steering wheel, accelerator and brake pedals, and dashboard, and placed on a 1 degree of freedom motion base responsive to pitch cues for braking and acceleration (See Figure 1). Three 80" projector screens surround the Honda Pilot for a $180^{\circ}$ field of view along with a rear view of the driving environment projected on a screen behind the

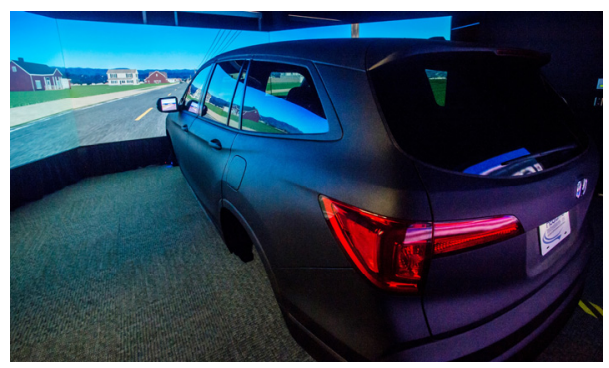

Figure 1. Driving simulator cab as well as in LCD screens in the side mirrors.

Module 1 - Basic Vehicle Operation. The first driving module included a bi-directional roadway in a daytime, rural setting with no ambient traffic.

Module 2 - Secondary Task. The same roadway and conditions as module 1, but with 2 secondary tasks (order randomized) while driving: 1) coin-sorting: participants were given amounts of change to count out for half of the module (Vickers, Schultheis, \& Manning, 2018); and 2) conversation: participants engaged in a naturalistic conversation via hands-free Bluetooth ${ }^{\circledR}$ with an unfamiliar research assistant for the other half of the module.

Module 3 - Car Following. Participants were instructed to follow and remain behind a lead vehicle for the entire module. The lead vehicle braked randomly, requiring a braking response from the participant, then resumed driving speed $(65 \mathrm{mph})$. The lead and participant vehicles remained in the right lane of a 4-land, divided highway with no turns and bidirectional traffic.

Module 4 -Divided Attention. Participants drove on a 4-lane, urban divided highway with bidirectional traffic and a speed limit of $55 \mathrm{mph}$. Ambient traffic travelled at $75 \mathrm{mph}$ and was dense 
across all lanes. Billboards containing images and text were placed on each side of the highway (side was randomized). These billboards were used previously in simulator studies (Stavrinos et al., 2016) and followed standard billboard regulations. Half of the billboards were randomized to have backwards text (i.e., mirrored left-to-right). Participants were instructed to press the cruise control button on the steering wheel as soon as they saw a billboard with backwards text.

Module 5 - Left-hand Turns Across Oncoming Traffic. Participants drove in an urban environment with a 4 lane, non-divided highway. Participants were instructed to turn left across oncoming traffic at 2 intersections. Oncoming vehicles were spaced with 4, 6, 8, or 12 second gaps between vehicles (gap order randomized). See Figure 2 for depiction of all driving modules.

Driving Outcomes. For all modules, average driving speed, speed variability (standard deviation of speed), and lane position variability (standard deviation of distance from center of lane) were calculated. Module 3 also included driver reaction time to the lead vehicle braking events.

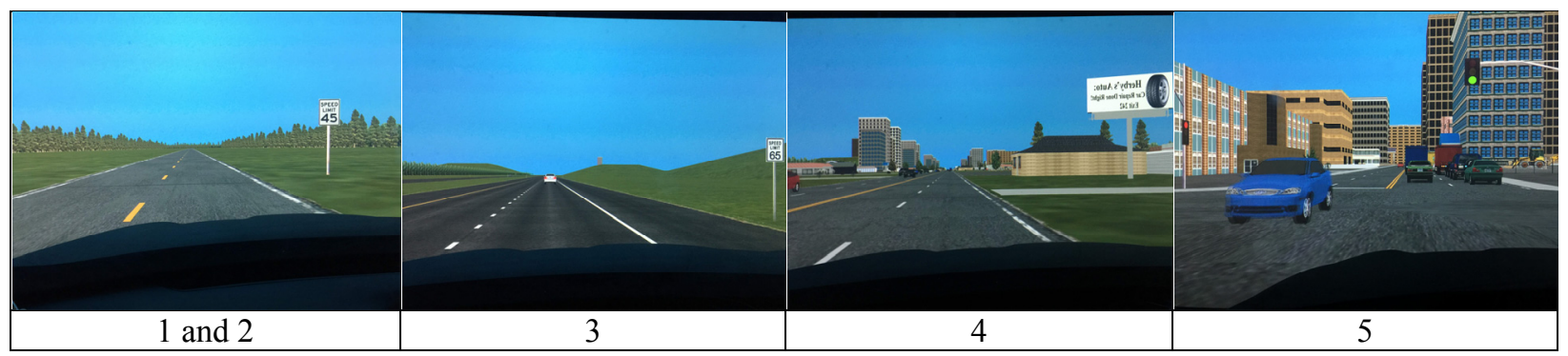

Figure 2. Driving modules

Cognitive Assessment. The Cogstate ${ }^{\mathrm{TM}}$ Brief Battery (Cogstate, 2017) was administered at the driving assessment appointment and utilized 4 computerized subtests: 1) Identification: measuring processing speed; 2) Detection: measuring attention; 3) One Card Learning: measuring visual learning; and 4) One Back: measuring working memory. The Cogstate ${ }^{\mathrm{TM}}$ Brief Battery has shown to be sensitive in detecting cognitive impairment in head injuries (Louey et al., 2014). Each subtest produced a score where higher values indicate increased correctly completed trials or responses as well as mean reaction time for all correct trials. Following all drives, participants completed the Brain Injury Driving Self-Awareness Measure (BIDSAM (Gooden et al., 2017)) to provide self-assessment of simulator driving difficulty.

Data Analyses. Differences on demographics, injury-related factors (i.e., time since injury), Cogstate outcomes, and driving outcomes for each module were assessed utilizing independent samples $t$-tests or non-parametric Wilcoxon Mann-Whitney $U$ tests, as appropriate. Bivariate Pearson correlations among all variables and driving outcomes for each module were conducted. Linear regression models were created to assess group differences on each module's driving outcomes while assessing demographic and injury-related variables (e.g., age, time since injury).

\section{RESULTS}

Active drivers were significantly older than non-drivers $(t(12)=2.21, p=.047$, Cohen's $d=$ 1.18). There were no differences between the groups of Cogstate performance, but marginal evidence suggested active drivers displayed faster identification $(U=68.00, p=.06)$ (Table 1).

Although no statistically significant differences were shown in driving outcomes between the two groups across the 5 driving modules (Table 2), in general, non-drivers displayed slower average driving speed and increased lane variation. Linear regressions with driver group, driver 
age, and time since injury produced a significant model predicting speed variability in Module 5 $\left(F=5.22, p=.03, R^{2}=.66\right)$, but there were no differences between the driving groups $(t=.63, p$ $=.54$, partial $\left.\eta^{2}=.05\right)$, and increased time since injury was most closely associated with speed variability $(t=3.53, p=.01)$. Although the regression model was not statistically significant in assessing lane position variability in Module $2\left(F=1.98, p=.18, R^{2}=.37\right)$, driver group was uniquely associated with lane variability such that non-drivers displayed significantly more variability compared to active drivers $\left(t=2.39, p=.04\right.$, partial $\left.\eta^{2}=.36\right)$.

Table 1. Demographic and Cogstate variables descriptive Statistics by driving group

\begin{tabular}{|c|c|c|c|c|c|c|c|}
\hline \multirow[t]{2}{*}{ Variable } & \multicolumn{3}{|c|}{ Non-Drivers } & \multicolumn{3}{|c|}{ Active Drivers } & \multirow[b]{2}{*}{ tor $U$} \\
\hline & Mean (SD) & $\mathrm{n}(\%)$ & Range & Mean (SD) & $\mathrm{n}(\%)$ & Range & \\
\hline Age (years) & $37.72(8.72)$ & & $24.26-50.74$ & $29.45(4.70)$ & & $20.67-34.76$ & 2.21 \\
\hline Gender (male) & & $5(71)$ & & & $4(57)$ & & $0.31^{\mathrm{a}}$ \\
\hline Race (White) & & $5(71)$ & & & $5(71)$ & & $0.00^{\mathrm{a}}$ \\
\hline Time Since Injury (years) & $2.80(2.09)$ & & $0.33-6.03$ & $4.66(3.52)$ & & $0.32-9.91$ & 1.20 \\
\hline Months Since Licensure & $256.80(105.90)$ & & $99.48-416.90$ & $157.90(59.65)$ & & $56.90-225.70$ & $2.15^{*}$ \\
\hline $\begin{array}{l}\text { BIDSAM } \\
\text { Cogstate Brief Battery }\end{array}$ & $20.14(5.43)$ & & $10.00-37.00$ & $17.86(7.13)$ & & $11.00-32.00$ & 0.55 \\
\hline Detection Score & $87.00(10.13)$ & & $78.00-102.00$ & $94.57(6.08)$ & & $85.00-100.00$ & 1.70 \\
\hline Detection Speed (ms) & $517.40(146.70)$ & & $316.00-685.00$ & $395.70(72.32)$ & & $334.00-508.00$ & 64.00 \\
\hline Identification Score & $88.71(8.38)$ & & $73.00-99.00$ & $95.57(11.13)$ & & $72.00-104.00$ & 1.30 \\
\hline Identification Speed (ms) & $759.40(185.30)$ & & $558.00-1141.00$ & $626.90(243.30)$ & & $473.00-1140.00$ & $68.00 *$ \\
\hline Learning Score & $97.57(7.50)$ & & $88.00-108.00$ & $99.71(10.40)$ & & $88.00-120.00$ & 0.44 \\
\hline Learning speed (ms) & $1691.40(431.20)$ & & $1208.00-2512.00$ & $1609.60(340.10)$ & & $1097.00-2143.00$ & 0.39 \\
\hline 1 Back Score & $86.57(6.35)$ & & $78.00-96.00$ & $87.14(6.64)$ & & $77.00-95.00$ & 0.16 \\
\hline 1 Back Speed (ms) & $1147.00(207.60)$ & & $877.00-1468.00$ & $1095.70(223.60)$ & & $8.38-1494.00$ & 0.44 \\
\hline
\end{tabular}

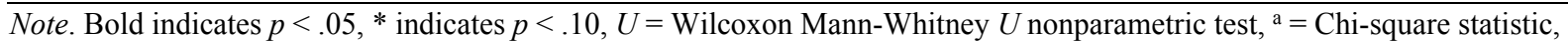
BIDSAM $=$ Brain Injury Driving Self-Awareness Measure, $\mathrm{ms}=$ millisecond

Table 2. Descriptive statistics for the 5 driving modules for non-drivers and active drivers

\begin{tabular}{|c|c|c|c|c|c|}
\hline \multirow{2}{*}{$\begin{array}{c}\text { Variable } \\
\text { Module } 1\end{array}$} & \multicolumn{2}{|c|}{ Non-Drivers } & \multicolumn{2}{|c|}{ Active Drivers } & \multirow[b]{2}{*}{ tor $U$} \\
\hline & Mean (SD) & Range & Mean (SD) & Range & \\
\hline Average Driving Speed (mph) & $42.80(4.11)$ & $36.42-47.80$ & $45.10(4.67)$ & $36.62-49.41$ & 0.98 \\
\hline Speed Variability (mph) & $6.24(1.71)$ & $4.13-8.73$ & $7.49(1.99)$ & $5.22-11.20$ & 1.26 \\
\hline Standard Deviation of Lane Position ( $\mathrm{ft}$ ) & $0.98(0.38)$ & $0.50-1.71$ & $0.99(0.23)$ & $0.77-1.47$ & 0.03 \\
\hline \multicolumn{6}{|l|}{ Module 2} \\
\hline Average Driving Speed (mph) & $41.53(5.53)$ & $32.37-47.50$ & $42.62(4.38)$ & $36.14-48.65$ & 0.41 \\
\hline Speed Variability (mph) & $9.28(4.84)$ & $3.75-15.44$ & $8.19(1.70)$ & $5.87-10.97$ & 0.56 \\
\hline Standard Deviation of Lane Position (ft) & $1.71(0.71)$ & $0.88-2.98$ & $1.27(0.35)$ & $0.89-1.85$ & 1.47 \\
\hline \multicolumn{6}{|l|}{$\underline{\text { Module } 3}$} \\
\hline Average Driving Speed (mph) & $51.73(8.57)$ & $35.58-59.38$ & $57.18(3.86)$ & $50.25-60.99$ & 1.53 \\
\hline Speed Variability (mph) & $10.95(1.50)$ & $8.68-12.90$ & $11.28(1.30)$ & $9.82-13.46$ & 0.45 \\
\hline Standard Deviation of Lane Position (ft) & $0.92(0.36)$ & $0.37-1.55$ & $0.75(0.30)$ & $0.45-1.33$ & 1.01 \\
\hline Average Braking Reaction Time (s) & $6.87(4.64)$ & $3.23-16.44$ & $3.97(1.74)$ & $2.35-7.32$ & 65.00 \\
\hline \multicolumn{6}{|l|}{ Module 4} \\
\hline Average Driving Speed (mph) & $52.36(2.74)$ & $48.86-57.58$ & $54.77(5.19)$ & $47.77-60.50$ & 1.05 \\
\hline Speed Variability (mph) & $10.32(2.03)$ & $7.66-14.20$ & $8.99(1.84)$ & $6.79-10.84$ & 1.16 \\
\hline Standard Deviation of Lane Position ( $\mathrm{ft}$ ) & $1.08(0.43)$ & $0.53-1.90$ & $0.85(0.18)$ & $0.66-1.12$ & 1.09 \\
\hline \multicolumn{6}{|l|}{ Module 5} \\
\hline Average Driving Speed (mph) & $32.22(3.04)$ & $29.16-37.50$ & $35.03(7.67)$ & $25.96-45.21$ & 0.89 \\
\hline Speed Variability (mph) & $11.88(1.87)$ & $10.10-15.31$ & $13.38(3.72)$ & $9.12-18.13$ & 0.92 \\
\hline Standard Deviation of Lane Position (ft) & $1.46(0.23)$ & $1.15-1.85$ & $1.44(0.30)$ & $1.08-1.74$ & 0.11 \\
\hline
\end{tabular}

Note. $\mathrm{mph}=$ miles per hours, $\mathrm{ft}=$ feet, and $\mathrm{s}=$ seconds, $U=$ Wilcoxon Mann-Whitney $U$ nonparametric test 
Correlations among variables and driving outcomes from module 1 in both groups are shown in Table 3, and correlations among variables and module 2-5 driving outcomes are shown for active drivers in Table 4, and non-active drivers Table 5. In active drivers but not in non-drivers, correlations suggest cognitive variables closely correlate with one another, particularly processing speed, attention, and working memory (Table 3 ). Under a potentially high cognitive load while driving (module 2, Tables 4-5), cognitive variables are more closely associated with driving outcomes in active drivers but not in non-drivers.

In the most complex modules (i.e., Module 5), processing speed and attention were more associated with driving outcomes in non-drivers, while working memory was more associated with driving in active drivers in general across all modules.

Table 3. Correlations among demographic, cognitive, and Module 1 driving outcomes

\begin{tabular}{|c|c|c|c|c|c|c|c|c|c|c|c|c|c|c|c|}
\hline \multirow[b]{2}{*}{ Module 1} & \multicolumn{3}{|c|}{ Driving } & \multicolumn{4}{|c|}{ Demographics and Injury } & \multicolumn{2}{|c|}{$\begin{array}{c}\text { Processing } \\
\text { Speed }\end{array}$} & \multicolumn{2}{|c|}{ Attention } & \multicolumn{2}{|c|}{$\begin{array}{c}\text { Visual } \\
\text { Learning }\end{array}$} & \multicolumn{2}{|c|}{$\begin{array}{l}\text { Working } \\
\text { Memory }\end{array}$} \\
\hline & 1 & 2 & 3 & 4 & 5 & 6 & 7 & 8 & 9 & 10 & 11 & 12 & 13 & 14 & 15 \\
\hline 1. $\quad$ Speed & & .08 & .14 & -.07 & -.19 & .18 & .11 & .17 & -.17 & -.08 & .13 & -.16 & -.79 & -.08 & .14 \\
\hline 2. SD Speed & .36 & & -.39 & -.21 & -.33 & -.52 & .34 & -.05 & .06 & -.31 & .28 & -.21 & -.44 & .17 & -.14 \\
\hline 3. SDLP & .54 & .51 & & .71 & .72 & .78 & .17 & -.44 & .46 & .14 & -.09 & -.00 & .06 & -.01 & .07 \\
\hline 4. $\quad$ Age & .29 & .08 & -.40 & & .98 & .66 & .14 & -.22 & .26 & .28 & -.20 & .09 & -.11 & .44 & -.35 \\
\hline 5. Licensure & .34 & .12 & -.38 & .99 & & .66 & .18 & -.22 & .27 & .32 & -.24 & .10 & .06 & .39 & -.32 \\
\hline 6. Time Since Injury & -.43 & .48 & -.40 & .16 & .20 & & .24 & -.59 & .61 & -.21 & .29 & .03 & -.06 & -.22 & .34 \\
\hline 7. BIDSAM & -.05 & -.80 & -.43 & .18 & .16 & -.52 & & -.77 & .77 & -.94 & .94 & -.33 & -.04 & -.58 & .69 \\
\hline 8. Detection & -.41 & -.17 & -.60 & -.21 & -.16 & .55 & -.12 & & -.99 & .77 & -.77 & .39 & -.22 & .63 & -.72 \\
\hline 9. Detection Speed & .48 & .19 & .46 & .44 & .39 & -.48 & .14 & -.97 & & -.75 & .75 & -.37 & .20 & -.59 & .68 \\
\hline 10. Identification & -.33 & .03 & -.20 & .51 & .49 & .30 & -.06 & -.29 & .35 & & -.99 & .54 & .05 & .77 & -.85 \\
\hline 11. ID Speed & .34 & -.05 & .05 & -.36 & -.35 & -.23 & .08 & .39 & -.40 & -.98 & & -.53 & -.11 & -.74 & .83 \\
\hline 12. Card Learning & -.37 & -.29 & -.70 & -.11 & -.10 & .38 & .31 & .78 & -.74 & -.46 & .55 & & -.11 & .60 & -.57 \\
\hline 13. CL Speed & -.19 & .07 & -.25 & -.22 & -.15 & .44 & .18 & .48 & -.53 & -.25 & .24 & .67 & & -.33 & .22 \\
\hline 14. One Back & -.03 & .32 & -.18 & .78 & .74 & .32 & -.10 & -.45 & .60 & .75 & -.69 & -.35 & -.31 & & -.98 \\
\hline 15. OB Speed & .12 & -.32 & .13 & -.67 & -.63 & .11 & .11 & .49 & -.60 & -.78 & .75 & .37 & .28 & -.96 & \\
\hline
\end{tabular}
Note: $=p<.05, \quad=p<.10, \quad=p<.20, \quad=p<.30, \quad=p<.40$. Non-drivers are represented below the diagonal and active drivers are represented above the diagonal. $\mathrm{SD}=$ Standard deviation, SDLP $=$ Standard deviation of lane position, $\mathrm{ID}=$ Identification, $\mathrm{CL}=$ Card Learning, and $\mathrm{OB}=$ One Back

Table 4. Correlations among demographics, cognitive, and driving in active drivers

\begin{tabular}{|c|c|c|c|c|c|c|c|c|c|c|c|c|}
\hline & \multicolumn{4}{|c|}{ Demographics and Injury } & \multicolumn{2}{|c|}{ Processing Speed } & \multicolumn{2}{|c|}{ Attention } & \multicolumn{2}{|c|}{ Visual Learning } & \multicolumn{2}{|c|}{ Working Memory } \\
\hline & Age & License & $\begin{array}{l}\text { Time } \\
\text { Since In- } \\
\text { jury }\end{array}$ & $\begin{array}{l}\text { BID- } \\
\text { SAM }\end{array}$ & Detect & $\begin{array}{l}\text { Detect } \\
\text { Speed }\end{array}$ & ID & $\begin{array}{c}\text { ID } \\
\text { Speed }\end{array}$ & $\mathrm{CL}$ & $\begin{array}{c}\text { CL } \\
\text { Speed }\end{array}$ & OB & $\begin{array}{c}\text { OB } \\
\text { Speed }\end{array}$ \\
\hline \multicolumn{13}{|l|}{ Module 2} \\
\hline Speed & -.16 & -.19 & .38 & .45 & -.71 & .69 & -.64 & .63 & -.43 & -.06 & -.72 & .76 \\
\hline SD Speed & .36 & .27 & .54 & .50 & -.67 & .68 & -.60 & .65 & -.61 & -.39 & -.38 & .49 \\
\hline SDLP & .72 & .74 & .91 & .06 & -.55 & .56 & -.10 & .17 & -.23 & .03 & -.22 & .30 \\
\hline \multicolumn{13}{|l|}{ Module 3} \\
\hline Speed & -.59 & -.69 & .21 & .07 & .28 & -.30 & -.19 & .19 & .15 & -.67 & -.13 & .16 \\
\hline SD Speed & .14 & .19 & -.09 & .46 & -.38 & .40 & -.20 & .17 & .20 & .41 & .13 & -.10 \\
\hline SDLP & .61 & .53 & .62 & -.23 & -.18 & .20 & .13 & -.07 & -.10 & -.46 & .11 & -.04 \\
\hline \multicolumn{13}{|l|}{ Module 4} \\
\hline Speed & -.65 & -.70 & -.03 & .56 & -.62 & .58 & -.75 & .69 & -.40 & .03 & -.94 & .93 \\
\hline SD Speed & .44 & .39 & .30 & .56 & -.34 & .37 & -.41 & .48 & -.33 & -.72 & .08 & .05 \\
\hline SDLP & .08 & .06 & -.43 & .04 & -.14 & .15 & .03 & -.09 & -.10 & .13 & .29 & -.32 \\
\hline \multicolumn{13}{|l|}{ Module 5} \\
\hline Speed & .07 & .10 & .65 & .02 & -.12 & .10 & -.14 & .21 & -.07 & -.10 & -.44 & .48 \\
\hline SD Speed & .13 & .14 & .68 & .17 & -.22 & .20 & -.28 & .35 & -.20 & -.17 & -.47 & .53 \\
\hline SDLP & .52 & .47 & .77 & .25 & -.70 & .71 & -.38 & .43 & -.57 & -.71 & -.34 & .44 \\
\hline
\end{tabular}

Note: $\mathrm{SD}=$ Standard deviation, SDLP $=$ Standard deviation of lane position, ID = Identification, $\mathrm{CL}=\mathrm{Card}$ Learning, and OB $=$ One Back 
PROCEEDINGS of the Tenth International Driving Symposium on Human Factors in Driver Assessment, Training and Vehicle Design

Table 5. Correlations among demographics, cognitive and driving in non-active drivers

\begin{tabular}{|c|c|c|c|c|c|c|c|c|c|c|c|c|}
\hline & \multicolumn{4}{|c|}{ Demographics and Injury } & \multicolumn{2}{|c|}{ Processing Speed } & \multicolumn{2}{|c|}{ Attention } & \multicolumn{2}{|c|}{ Visual Learning } & \multicolumn{2}{|c|}{ Working Memory } \\
\hline & Age & License & $\begin{array}{l}\text { Time } \\
\text { Since } \\
\text { Injury }\end{array}$ & $\begin{array}{l}\text { BID- } \\
\text { SAM }\end{array}$ & Detect & $\begin{array}{l}\text { Detect } \\
\text { Speed }\end{array}$ & ID & $\begin{array}{c}\text { ID } \\
\text { Speed }\end{array}$ & $\mathrm{CL}$ & $\begin{array}{c}\text { CL } \\
\text { Speed }\end{array}$ & OB & $\begin{array}{c}\text { OB } \\
\text { Speed }\end{array}$ \\
\hline Module 2 & & & & & & & & & & & & \\
\hline Speed & 0.1 & 0.09 & 0.06 & 0.29 & -0.03 & 0.07 & -0.5 & 0.51 & 0.54 & 0.52 & 0.03 & -0.02 \\
\hline SD Speed & -0.08 & -0.03 & 0.28 & -0.6 & -0.36 & 0.3 & 0.09 & -0.18 & -0.42 & 0.22 & 0.19 & -0.24 \\
\hline SDLP & -0.59 & -0.54 & -0.17 & -0.27 & -0.29 & 0.11 & -0.3 & 0.13 & -0.3 & 0.33 & -0.41 & 0.33 \\
\hline Module 3 & & & & & & & & & & & & \\
\hline Speed & 0.41 & 0.35 & 0.41 & 0 & 0.43 & -0.26 & 0.14 & 0.02 & 0.45 & -0.22 & 0.38 & -0.32 \\
\hline SD Speed & 0.35 & 0.41 & 0.15 & 0.05 & -0.2 & 0.26 & -0.12 & 0.12 & 0.06 & 0.54 & 0.18 & -0.14 \\
\hline SDLP & -0.39 & -0.37 & -0.36 & -0.35 & -0.62 & 0.48 & -0.25 & 0.09 & -0.59 & -0.09 & -0.16 & 0.1 \\
\hline Module 4 & & & & & & & & & & & & \\
\hline Speed & 0.34 & 0.32 & -0.15 & -0.54 & -0.44 & 0.51 & 0.3 & -0.28 & -0.78 & -0.83 & 0.4 & -0.34 \\
\hline SD Speed & 0.42 & 0.48 & 0.13 & -0.56 & -0.04 & 0.17 & 0.04 & 0.03 & -0.4 & -0.3 & 0.18 & -0.07 \\
\hline SDLP & -0.6 & -0.6 & -0.39 & -0.35 & -0.55 & 0.36 & -0.28 & 0.09 & -0.54 & -0.12 & -0.27 & 0.18 \\
\hline Module 5 & & & & & & & & & & & & \\
\hline Speed & -0.08 & -0.02 & 0.23 & -0.58 & 0.41 & -0.35 & -0.59 & 0.66 & 0.19 & 0.08 & -0.33 & 0.42 \\
\hline SD Speed & 0.15 & 0.21 & 0.95 & -0.41 & 0.54 & -0.49 & 0.23 & -0.17 & 0.41 & 0.65 & 0.21 & -0.22 \\
\hline SDLP & -0.39 & -0.35 & -0.16 & -0.58 & -0.43 & 0.31 & -0.24 & 0.11 & -0.56 & -0.07 & -0.18 & 0.13 \\
\hline
\end{tabular}

Note: $\mathrm{SD}=$ Standard deviation, $\mathrm{SDLP}=$ Standard deviation of lane position, ID = Identification, $\mathrm{CL}=\mathrm{Card}$ Learning, and $\mathrm{OB}=$ One Back.

\section{CONCLUSION}

This study was among the most recent works to utilize a high-fidelity driving simulator to determine its potential in informing return to driving decisions, contributing to previous support in the use of driving simulators when deciding post-TBI return-to-driving (Lew et al., 2005; Lew et al., 2009). Although no statistically significant differences between active drivers and non-drivers were found, several driving outcomes trend towards indicating group differences. It is possible the modules were not sufficiently sensitive towards differences between the two groups. The small sample size of $n=7$ per group is a limitation, but this study provided valuable insight into the cognitive processes that may be differentially associated with driving situations that are ethically and/or experimentally impossible replicate in a real-world on-road driving assessment. The assessment of key cognitive factors and how they relate to specific driving variables is valuable when cognition is highly impacted as a result of the injury itself. In the active drivers, as driving complexity increased (i.e., from module-to-module), higher order cognitive processes were more associated with driving outcomes, suggesting convergent validity of the driving modules. These preliminary findings inform future development of driving modules. The modules involving specific tasks beyond basic vehicle navigation provide the most potential for sensitivity between driving groups. Modules with little navigation requirements (i.e., no turns, or curve) displayed trending differences in reaction time, speed, and vehicle position maintenance. Future development of these modules may include more complex driving, such as tasks involving specific domains of attention during the driving task (e.g., selective attention).

\section{ACKNOWLEDGEMENTS}

This work was supported by funding from the UAB Department of Physical Medicine and Rehabilitation Resident Physician Collaborative Research in Functional Neurorecovery Award. The UAB Edward R. Roybal Center for Translational Research in Aging and Mobility (5 P30 AG 022838 PI: Karlene K. Ball) and Translational Research for Injury Prevention (TRIP) Laboratory provided lab space and the driving simulator. Special thanks to Dr. Thomas Novack from the 
UAB TBI Clinic for participant recruitment and group classification, and to TRIP lab assistants for recruitment and data collection and entry. The authors also gratefully thank Dr. Andrea Underhill for providing feedback on an earlier version of this manuscript.

\section{REFERENCES}

Cogstate. (2017). Cogstate Brief Battery. New Haven, CT: Cogstate Healthcare.

Edquist, J., Horberry, T., Hosking, S., \& Johnston, I. (2011). Effects of advertising billboards during simulated driving. Applied Ergonomics, 42(4), 619-626. doi:10.1016/j.apergo.2010.08.013

Gooden, J. R., Ponsford, J. L., Charlton, J. L., Ross, P. E., Marshall, S., Gagnon, S., . . Stolwyk, R. J. (2017). The development and initial validation of a new tool to measure self-awareness of driving ability after brain injury. Australian Occupational Therapy Journal, 64(1), 33-40. doi:10.1111/1440-1630.12306

Jahn, G., Oehme, A., Krems, J. F., \& Gelau, C. (2005). Peripheral detection as a workload measure in driving: Effects of traffic complexity and route guidance system use in a driving study. Transportation Research Part F: Psychology and Behaviour, 8(3), 255-275.

Lew, H. L., Poole, J. H., Lee, E. H., Jaffe, D. L., Huang, H. C., \& Brodd, E. (2005). Predictive validity of driving-simulator assessments following traumatic brain injury: a preliminary study. Brain Injury, 19(3), 177-188.

Lew, H. L., Rosen, P. N., Thomander, D., \& Poole, J. H. (2009). The potential utility of driving simulators in the cognitive rehabilitation of combat-returnees with traumatic brain injury. Journal of Head Trauma Rehabilitation, 24(1), 51-56. doi:10.1097/HTR.0b013e3181956fe3

Louey, A. G., Cromer, J. A., Schembri, A. J., Darby, D. G., Maruff, P., Makdissi, M., \& McCrory, P. (2014). Detecting cognitive impairment after concussion: sensitivity of change from baseline and normative data methods using the CogSport/Axon cognitive test battery. Archives of Clinical Neuropsychology, 29(5), 432-441. doi:10.1093/arclin/acu020

National Highway Traffic Safety Administration. (2015). Traffic safety facts: Critical reasons for crashes investigated in the national motor vehicle crash causation survey. (DOT HS 812 115). Washington, DC: NHTSA's National Center for Statistics and Analysis Retrieved from http://www-nrd.nhtsa.dot.gov/pubs/812115.pdf.

Stavrinos, D., Mosley, P. R., Wittig, S. M., Johnson, H. D., Decker, J. S., Sisiopiku, V., \& Welburn, S. C. (2016). Visual behavior differences in drivers across the lifespan: A digital billboard simulator study. Transportation Research Part F: Traffic Psychology and Behaviour, 41, 19-28. doi:10.1016/j.trf.2016.06.001

Vickers, K. L., Schultheis, M. T., \& Manning, K. J. (2018). Driving after brain injury: Does dual-task modality matter? NeuroRehabilitation, 42(2), 213-222. doi:10.3233/nre-172301

Xu, J., Murphy, S. L., Kochanek, K. D., \& Bastian, B. A. (2016). Deaths: Final data for 2013 (Vol. 64): Centers for Disease Control and Prevention. 